University of Nebraska - Lincoln

DigitalCommons@University of Nebraska - Lincoln

Publications from USDA-ARS / UNL Faculty

U.S. Department of Agriculture: Agricultural

Research Service, Lincoln, Nebraska

$1-1-2021$

\title{
Pheromone antagonism in Plutella xylostella (Linnaeus) by sex pheromones of two sympatric noctuid moths
}

\author{
Fu Min Wang \\ Zhejiang Agriculture and Forestry University \\ Zhi Jie Shen \\ Zhejiang Agriculture and Forestry University \\ Coby Schal \\ NC State University \\ Junwei J. Zhu \\ USDA-ARS Agroecosystem Management Research Unit, USDA-ARS, University of Nebraska, East Campus, \\ jerry.zhu@ars.usda.gov \\ Guo Xin Zhou \\ Zhejiang Agriculture and Forestry University
}

See next page for additional authors

Follow this and additional works at: https://digitalcommons.unl.edu/usdaarsfacpub

Part of the Agriculture Commons

Wang, Fu Min; Shen, Zhi Jie; Schal, Coby; Zhu, Junwei J.; Zhou, Guo Xin; Wang, Yu Lei; Wang, Ji Rui; Deng, Jian Yu; and Wan, Nian Feng, "Pheromone antagonism in Plutella xylostella (Linnaeus) by sex pheromones of two sympatric noctuid moths" (2021). Publications from USDA-ARS / UNL Faculty. 2511. https://digitalcommons.unl.edu/usdaarsfacpub/2511

This Article is brought to you for free and open access by the U.S. Department of Agriculture: Agricultural Research Service, Lincoln, Nebraska at DigitalCommons@University of Nebraska - Lincoln. It has been accepted for inclusion in Publications from USDA-ARS / UNL Faculty by an authorized administrator of DigitalCommons@University of Nebraska - Lincoln. 


\section{Authors}

Fu Min Wang, Zhi Jie Shen, Coby Schal, Junwei J. Zhu, Guo Xin Zhou, Yu Lei Wang, Ji Rui Wang, Jian Yu Deng, and Nian Feng Wan 


\title{
Pheromone antagonism in Plutella xylostella (Linnaeus) by sex pheromones of two sympatric noctuid moths
}

\author{
Fu-Min Wang, ${ }^{\text {a,b }}$ Zhi-Jie Shen, ${ }^{a}$ Coby Schal, ${ }^{c}$ Junwei J Zhu, ${ }^{d}$ Guo-Xin Zhou, ${ }^{a}$ \\ Yu-Lei Wang, ${ }^{a}$ Ji-Rui Wang, ${ }^{a}$ Jian-Yu Deng ${ }^{a^{*}} \odot$ and Nian-Feng Wan ${ }^{e^{*}} \odot$
}

\begin{abstract}
BACKGROUND: Responses to sex pheromones are commonly antagonized by pheromone components of closely related species. Pheromone antagonism has not been widely explored for phylogenetically distant species that have completely different pheromone components. Yet, pheromone components of sympatrically occurring species may also interfere with each other even if these species are distantly related. Here, the effects of heterospecific pheromones on electrophysiology (electroantennogram, EAG) and behavioral responses were tested on the diamondback moth Plutella xyloslella (Plutellidae) and two sympatric noctuid moth species, Spodoptera litura and Spodoptera exigua, whose larvae also feed on Brassica crops.
\end{abstract}

RESULTS: The sex pheromone blend of $P$. xyloslella, and its components, did not elicit EAG responses in males of the two noctuid species, while sex pheromone components of the noctuid moths elicited significant EAG responses in $P$. xyloslella males. In wind tunnel bioassays, both $(Z, E)-9,12$-tetradecadienyl acetate $(Z E-9,12-14: O A C)$ and $(Z, E)-9,11$-tetradecadienyl acetate (ZE-9,11-14: $O A c)$, sex pheromone components from the noctuid moths, inhibited the upwind flight behavior of $P$. xyloslella males toward an intraspecific pheromone odor source. In Brassica fields, sex pheromone lures of $P$. xyloslella did not influence trap catches of the noctuid moths, while $P$. xyloslella pheromone lures baited with either ZE-9,11-14:OAc or ZE-9,12-14:OAc decreased trap catches of $P$. xyloslella males in a dose-dependent manner. Trap catches of $P$. xylostella males were also affected by the proximity of ZE-9,11-14:OAC or ZE-9,12-14:OAC to $P$. xylostella lures.

CONCLUSION: The uni-directional pheromone antagonism by ZE-9,11-14:OAC and ZE-9,12-14:OAC suggests innovative semiochemical-based strategies for the management of $P$. xyloslella and other economically important pests in Brassica fields. () 2021 Society of Chemical Industry.

Supporting information may be found in the online version of this article.

Keywords: pheromone antagonism; antennal response; Plutella xyloslella; mating disruption; sympatry; semiochemical

\section{INTRODUCTION}

Reproduction in moth species relies heavily on sex pheromones released by females that attract conspecific males. Sex pheromones are species-specific and are often emitted as a blend of multiple components that collectively enable conspecific matefinding while preventing infertile hybrids from cross-mating. ${ }^{1,2}$ However, under some conditions, species-specific pheromones might fail to guide conspecific males to the odor source due to the presence of confounding sensory cues in their habitat, including heterospecific sex pheromone compounds released by other species with overlapping temporal and spatial sexual communication. ${ }^{3,4}$ Such overlap, and potential interference in sexual communication, are expected to occur in agricultural pests whose own phenologies are guided by crop phenology. Also, invasive species that are allopatric in their respective native habitat may interfere with each other in their new sympatric habitat.

For sympatric moth species, interspecific interference of sexual communication is generally avoided by either temporal separation of calling behavior or divergence of their pheromone
"Correspondence to: J-Y Deng, Department of Plant Protection, College of Advanced Agricultural Sciences, Zhejiang A \& F University, N0.666, Wusu Street, Lin'an district, Hangzhou, Zhejiang, 311300, China, E-mail: jydeng70@aliyun. com (Deng); or N-F Wan, Eco-environmental Protection Research Institute, Shanghai Academy of Agricultural Sciences, Shanghai Key Laboratory of Protected Horticultural Technology, N0.1000,Jinqi Road, Fengxian district, Shanghai, 201403, China. E-mail: fnianwan_2004@163.com (Wan)

a Department of Plant Protection, College of Advanced Agricultural Sciences, Zhejiang A \& F University, Wusu Street, Hangzhou, Zhejiang, 311300, China

b Division of Plant and Soil Sciences, West Virginia University, Morgantown, WV, USA

c Department of Entomology and W. M. Keck Center for Behavioral Biology, North Carolina State University, Raleigh, NC, USA

d USDA-ARS, Agroecosystem Management Research Unit, Lincoln, NE, USA

e Eco-Environmental Protection Research Institute, Shanghai Academy of Agricultural Sciences, Shanghai Key Laboratory of Protected Horticultural Technology, Jinqi Road, Shanghai, 201403, China 
blends. ${ }^{5}$ While it is common for sibling species to share major pheromone components due to common ancestry, distantly related species (different genera) may also release the same pheromone compounds because moths share similar pheromone biosynthetic pathways in their pheromone glands. ${ }^{6}$ Females generally produce 'minor' components, which maintain speciesspecificity, and the male antennae express pheromone receptors specifically tuned to each of the pheromone components of the female blend. ${ }^{7-10}$ These components are usually geometric isomers or analogs of the common major pheromone compound, and they share biosynthetic pathways. The species-specific minor components and the overall blend ratio are often detected by heterospecific males and cause these males to cease their pheromone-guided anemotaxis. ${ }^{9}$ There are also a few cases in which pheromone antagonism occurs between sympatric moths that share no common pheromone compounds. These species are often distantly related phylogenetically and belong to different genera ${ }^{4,11,12}$ or more rarely to different families. ${ }^{3,13}$

In this study, we focused on two different families of moth pests that attack Brassica plants including a variety of agricultural and horticultural crops. In China, significant economic damage to Brassica production is caused by spatial-temporal co-occurrences of multiple herbivores including larvae of the oligophagous moth Plutella xylostella Linnaeus (Lepidoptera: Plutellidae) and two polyphagous moths, Spodoptera litura Fabricius (Lepidoptera: Noctuidae) and Spodoptera exigua Fabricius (Lepidoptera: Noctuidae). The pheromone blends of $P$. xylostella and the two noctuid moths do not share any pheromone components. For each species, the major functional components have been well characterized and applied to species-specific mass trapping and surveillance programs in China (summarized in Table 1). Females of $P$. xylostella release (Z)-11-hexdecenyl acetate (Z11-16:OAC), (Z)-11-hexadecenal (Z11-16:Ald), and (Z)-11-hexadecenol (Z11-16:OH), and a blend ratio of these respective components at 27:64:9 (denoted as pheromone blend $\mathrm{Px}$ ) at $10 \mu \mathrm{g}$ was strongly attractive to conspecific males in China. ${ }^{14,15}$ In contrast, extracts from pheromone glands of these two noctuid moths ( $S$. litura and S. exigua) contain mainly 14-carbon alcohol, aldehyde, and acetate esters. ${ }^{16,17}$ In China, highly attractive pheromone blends for S. exigua ${ }^{16}$ contain $(Z, E)-9,12$-tetradecadienyl acetate (ZE-9,12-14:OAC) and (Z)-9-tetradecenol (Z9-14:OH) (pheromone blend $\mathrm{Se}, 90: 10 \mathrm{w} / \mathrm{w}$ ) at $100 \mu \mathrm{g}$ and blends for S. litura ${ }^{18}$ contain $(Z, E)-9,11$-tetradecadienyl acetate (ZE-9,11-14:OAc) and ZE-9,12-14:OAC (pheromone blend SI, 90:10 w/w) at $100 \mu \mathrm{g}$.

As larvae of $P$. xylostella and the two noctuid moths occur sympatrically on Brassica crops, in this study we hypothesized that: (i) the antennae of females and males of each of the three species would respond to different amounts of heterospecific sex pheromone components; (ii) pheromone antagonism would occur between $P$. xylostella and these two noctuid moths; (iii) pheromone antagonism might be effective in the field and therefore potentially useful in pest management. To test our hypotheses, we first measured the electrophysiological responses of female and male antennae of each species to each heterospecific sex pheromone blend. Because only male antennae of P. xylostella responded to sex pheromone components of the noctuid moths, we evaluated the effects of noctuid pheromones on pheromone-guided behavior of $P$. xylostella in a wind tunnel. In a cabbage (Brassica oleracea L.) field, we assessed the attraction of males of the three species to their species-specific lures with and without heterospecific components. The attraction of $P$. xylostella males to sex pheromone traps was also examined in a cauliflower ( $B$. oleracea var. botrytis) field by combining lures with different doses of sex pheromones of the noctuid moths. In another cabbage field ( $B$. oleracea) sex pheromone traps were arranged at various distances from a noctuid pheromone lure to assess the effects of distance from the antagonistic pheromone on trap captures. The results of this study improve our understanding of the function of sex pheromones in sympatric moth species. This study also provides insights into the mechanisms by which pheromone antagonism might enhance the biological control of multiple species of insect herbivores in their shared host crops.

\section{MATERIALS AND METHODS}

\subsection{Pheromone chemicals}

Synthetic Z11-16:OAC (CAS: 34010-21-4), Z11-16:Ald (CAS: 53939-28-9), Z11-16:OH (CAS: 56683-54-6), ZE-9,11-14:OAC (CAS: 50767-79-8), ZE-9,12-14:OAC (CAS: 30507-70-1) and Z9-14:OH (CAS: 35153-15-2) were purchased from Shin-Etsu Chemical Co., Ltd. All chemicals were verified to $>97 \%$ purity by GC-MS before experiments.

\subsection{Insects}

Laboratory populations of $P$. xylostella and the two noctuid moths (S. litura and S. exigua) used for electrophysiological recordings originated from adults collected from different cabbage fields all of which were free from pesticide application. All three species were maintained under $14 \mathrm{~L}: 10 \mathrm{D}$ at $25 \pm 2{ }^{\circ} \mathrm{C}$ and $70 \%$ relative humidity, and they were reared by methods adapted from previous studies. ${ }^{19,20}$ Adults of $P$. xylostella were obtained by rearing larvae on cabbage plants in large plastic-screen cages $(3.5 \times 3.5 \times 1.5 \mathrm{~m})$. Pupae were removed to another cage $(0.5 \times 0.5 \times 1 \mathrm{~m})$ and newly emerged adults were separated into individual test tubes containing cotton pads impregnated with approximately $10 \%$ honey in water. Adults of S. litura and $S$. exigua were placed in separate large plastic-screen cages $(3.5 \times 3.5 \times 1.5 \mathrm{~m})$, supplemented with $10 \%$ honey in water, and

Table 1. Composition of pheromone blends of three moth species used in this study

\begin{tabular}{|c|c|c|c|c|c|c|c|}
\hline \multirow[b]{2}{*}{ Species } & \multirow[b]{2}{*}{ Pheromone blend } & \multicolumn{6}{|l|}{ Proportion $(\%)^{\dagger}$} \\
\hline & & ZE-9,11-14:OAC & ZE-9,12-14:OAC & Z9-14:OH & $\mathrm{Z} 11-16: \mathrm{OAC}$ & Z11-16:Ald & $\mathrm{Z11-16:OH}$ \\
\hline Spodoptera litura & SI & 90 & 10 & - & - & - & - \\
\hline Spodoptera exigua & $\mathrm{Se}$ & - & 90 & 10 & - & - & - \\
\hline Plutella xylostella & Px & - & - & - & 27 & 64 & 9 \\
\hline
\end{tabular}


stencil paper was placed inside for egg deposition. Eggs were transferred into new cages containing an artificial diet for larvae and coarse soil for pupation. ${ }^{21}$ Pupae were transferred to jars each containing a moistened paper at the base, and newly emerged adults were individually transferred to test tubes supplemented with cotton pads impregnated with approximately $10 \%$ honey in water.

\subsection{Electroantennogram recordings}

At the onset of scotophase, unmated 2 to 3-day-old males and females of the three moth species were acclimatized under the experimental conditions. All tests were conducted $4-8 \mathrm{~h}$ from the onset of scotophase. An antenna from each $\mathrm{CO}_{2}$-anaesthetized adult was detached at the base with a dissecting scalpel and attached to an adjustable antenna holder (steel electrode) supported by electrically conductive gel. The electrode was supported on an MP-15 micromanipulator covered with a steel mesh screen that was connected to the ground of an electrical outlet. The amplified signal was captured by the signal acquisition interface IDAC-2 (Syntech) connected to a computer. A stimulus controller (CS-55; Syntech) was used to deliver constant humidified air at $5 \mathrm{~mL} \mathrm{~s}{ }^{-1}$ over the antenna through a $7 \mathrm{~mm}$ diameter Teflon tube. The CS-55 was also used to generate an independent air flow $\left(0.5 \mathrm{~s}, 4 \mathrm{~mL} \mathrm{~s}^{-1}\right)$ that delivered the stimulus odor as a pulse into a steel mixing tube that terminated $1 \mathrm{~cm}$ from the antenna. To test antennal responses of both sexes of each moth species to each sex pheromone blend, all sex pheromone blends were dissolved in hexane to four concentrations $\left(0,0.1,1\right.$, and $\left.10 \mu \mathrm{g} \mathrm{LL}^{-1}\right)$. To test the antennal responses of $P$. xylostella males to conspecific and heterospecific sex pheromone components, each component was dissolved in hexane and diluted to three concentrations $\left(0.1,1\right.$, and $\left.10 \mu \mathrm{g} \mathrm{L}^{-1}\right)$. In both experiments, $10 \mu \mathrm{L}$ of each solution was pipetted onto a rectangular filter paper $(1 \times 3 \mathrm{~cm})$, and the paper was then inserted into a Pasteur pipette within 5 min of loading the stimulus. The wide section of the Pasteur pipette was connected to the pulse flow tube and the narrow end was inserted through a hole in the steel mixing tube. In the first experiment, each antenna was stimulated by only one sex pheromone blend with increasing concentrations and each concentration was tested with three puffs $30 \mathrm{~s}$ apart. In the second experiment, each antenna was stimulated by one dose in the following order: ZE-9,11-14:OAC, ZE-9,12-14:OAC, Z9-14:OH, Z11-16:Ald, Z11-16:OAC, and $Z 11-16: O H$. Each component was tested with three successive puffs. In both experiments, EAG values $(\mathrm{mV})$ from the three puffs for each treatment (pheromone blend/compound $\times$ concentration) were averaged, and six antennae $(n=6)$ were used as replicates for each treatment. The Pasteur pipette and filter paper stimulus were replaced with a fresh stimulus after each antenna replicate.

\subsection{Wind tunnel bioassay}

Since antennae of $P$. xylostella males could detect the sex pheromones of the two Spodoptera species, the upwind flight of $P$. xylostella males to its species-specific pheromone odor source (Px) in the presence of the noctuid pheromones was investigated in a cylindrical wind tunnel apparatus (diameter, $50 \mathrm{~cm}$; length, $180 \mathrm{~cm}$; illustrated in Fig. S1) under $5 \mathrm{~lx}$ red light illumination at $27 \pm 2{ }^{\circ} \mathrm{C}$ and $70-80 \%$ relative humidity. Air velocity of approximately $40 \mathrm{~cm} \mathrm{~s}^{-1}$ was generated by a fan at the downwind end of the tunnel. To prepare odor sources, different pheromone blends or components were dissolved in hexane and applied to a common rectangular piece of filter paper $(5 \times 7 \mathrm{~cm})$, which was then transferred into a cylindrical wire screen cage $(O D, 6 \mathrm{~cm}$; height, $10 \mathrm{~cm}$ ) $5 \mathrm{~min}$ after pheromone application. Filter papers with sex pheromones were replaced after three replicate trials. Unmated 2-day-old males were individually transferred to glass test tubes $(\mathrm{OD}, 3 \mathrm{~cm}$; height, $10 \mathrm{~cm})$ and acclimated to the wind tunnel conditions 60 min before tests. Each male was tested only once by being released $120 \mathrm{~cm}$ downwind of the odor source and all males were tested 4-8 $\mathrm{h}$ into scotophase. The internal surface of the wind tunnel and the screen cage was washed with hexane after 10 replicate trials. Each male was tested for no more than $8 \mathrm{~min}$ and the behavioral responses were categorized as 'take-off' (TO), oriented flight to half the length of the tunnel $(H T)$, approached the source within $15 \mathrm{~cm}$ of the lure $(A P)$, and source contact (SC).

\subsection{Field trapping experiments}

In all field experiments, red plastic basins (ID, $20 \mathrm{~cm}$ ) filled with dilute detergent solution were used as traps. Lures were prepared by loading pheromone blends or components into red cylinder rubber septa (ID, $10 \mathrm{~mm}$; height, $10 \mathrm{~mm}$; Institute of Plant Physiology \& Ecology, Shanghai Institutes for Biological Sciences, Chinese Academy of Sciences). A metal wire was used to tie a single lure or two lures together $2 \mathrm{~cm}$ above the water surface.

The first field trapping experiment was conducted in a cabbage field in Taizhou City, China, from 26 May to 3 June 2013. The attraction of each of the three moth species was evaluated according to their species-specific lures or combined lures at doses optimized for each species. ${ }^{15,16,18}$ As a follow-up, a second trapping period was carried out in a cauliflower field in Shanghai, China, from 4-12 June 2014 to assess the dose-response effects of the noctuid moth sex pheromones on the attraction of $P$. xylostella males to the Px pheromone blend. In these two field experiments, each treatment was replicated with six traps $(n=6)$. Hexane-loaded septa were used as a negative control. Traps were placed 6-7 m apart, with randomly assigned treatment positions. Moth species were identified, counted, and removed daily. Traps were re-randomized daily to minimize positional effects.

A third trapping experiment was conducted in three adjacent cabbage fields in Pinghu County, Zhejiang, China, from 22-28 October 2017 to determine the effect of the distance of ZE-9,11-14:OAC and ZE-9,12-14:OAC lures on the attraction of $P$. xylostella males to the Px pheromone blend. This experiment was conducted when the field population of $P$. xylostella was declining. In each of the three fields, 25 basin traps, each baited with a rubber septum impregnated with $10 \mu \mathrm{g}$ Px pheromone, were evenly arranged in a $5 \times 5$ layout (Fig. 5(A)). Traps were placed $2 \mathrm{~m}$ apart and $15 \mathrm{~cm}$ above the plant canopy (Fig. 5(A)). The central trap in each trap array was baited with an additional rubber septum loaded with either hexane (control, CK), ZE9,11-14:OAC $(100 \mu \mathrm{g})$, or ZE-9,12-14:OAC $(100 \mu \mathrm{g})$, and tied together with the Px pheromone lure. By this design, captures of $P$. xylostella males were expected to be suppressed according to the distance from the central trap (Fig. 5(A)). Captures of $P$. xylostella males at each trapping site were counted, recorded, and removed daily and all traps except the central trap were re-randomized daily.

\subsection{Data analysis}

The antennal responses of females and males of $P$. xylostella to the pheromone blends of the noctuid moths were analyzed with a general linear model (GLM) explaining the effects of the pheromone blend, pheromone dose, moth sex, and their binary and ternary interactions. We selected the GLM based on the Akaike information criterion (AIC) using the aictab function in the 
'AICcmodavg' package. ${ }^{22}$ The antennal responses of the two noctuid moths to the pheromone blend of $P$. xylostella were analyzed in separate GLMs with the effects of pheromone dose, moth sex, and their interaction. The antennal responses of $P$. xylostella males to conspecific and heterospecific sex pheromone components were analyzed in a GLM analyzing the effects of component, pheromone dose, and their interaction. All model effects from EAG experiments were analyzed by ANOVA $(\alpha=0.05)$ and estimated marginal means (emmeans) were compared with $p$-values adjusted by Tukey's HSD tests. In the wind tunnel bioassay, the percentages of $P$. xylostella males that showed a behavioral response (i.e., TO, HT, AP or SC) out of the total males tested were compared among different pheromone sources using a binomial generalized linear model (GLIM) with a logit link. The emmeans were compared using Tukey's HSD for $P$-value adjustment. We considered the hexane control as an independent negative control and did not include it in the multiple comparisons. In the first two field experiments, the effect of pheromone lures on total captures of each species was identified by a likelihood-ratio Chi-squared test (Type II ANOVA, $\alpha=0.05$ ) from a Poisson GLIM with a log link, and Tukey's HSD tests were used to compare emmeans. In the third field trapping experiment, the effects of distance $(X)$ of $P x$ pheromone-baited traps from the central trap (baited with two lures loaded with, respectively, Px and one pheromone component of the noctuid moths) on total captures $(\mathrm{Y})$ of $P$. xylostella males were determined in separate Poisson GLIMs [i.e., $\left.\log (\mathrm{Y})=\beta_{0}+\beta_{1} \mathrm{X}\right)$ ] by analyzing the estimated coefficients of 'distance' $\left(\beta_{1}\right)$ with one-sample $z$-tests $(\alpha=0.05)$. All the procedures were programmed in $\mathrm{R}$ (version 3.4.3; R Development Core Team, www.R-project.org). ${ }^{23}$

\section{RESULTS}

\subsection{P. xylostella males olfactorily detect noctuid moth pheromones}

The antennal responses in these two noctuid moth species to sex pheromone blend of $P$. xylostella (Px) were consistently low and did not vary significantly with moth sex (S. litura: $F_{1,40}=0.001$, $P=0.970$; S. exigua: $F_{1,40}=0.001, P=0.982$ ), different pheromone doses (S. litura: $F_{3,40}=0.097, P=0.961$; S. exigua: $F_{3,40}=0.059$, $P=0.981$ ), or their interaction (S. litura: $F_{3,40}=0.125, P=0.944$; S. exigua: $\left.F_{3,40}=0.140, P=0.935\right)$. However, the antennal responses of $P$. xylostella to the pheromone blends of the noctuid moths varied significantly with moth sex $\left(F_{1,128}=330.246, P<0.001\right)$, noctuid pheromone blends $\left(F_{1,128}=98.335, P<0.001\right)$, doses of pheromone blend $\left(F_{3,128}=134.956, P<0.001\right)$, all their binary interactions (sex$x$ blend: $F_{1,128}=32.392, P<0.001$; sex $\times$ dose: $F_{3,128}=69.118$, $P<0.001$; blend $\times$ dose: $\left.F_{3,128}=24.345, P<0.001\right)$ as well as the ternary interaction (sex $\times$ blend $\times$ dose: $F_{3,128}=11.591$, $P<0.001$ ). By multiple comparisons (Tukey's HSD) it was found that the antennal responses in female $P$. xylostella did not vary significantly to various doses of pheromone blend $\mathrm{Se}$, or to various doses of pheromone blend SI $(P>0.05)$. However, compared to the hexane control, significantly higher EAG responses were elicited in males of $P$. xylostella by $\geq 10 \mu \mathrm{g}$ pheromone blend $\mathrm{SI}(t=5.830$, $P<0.001)$ and by $\geq 1 \mu \mathrm{g}$ pheromone blend Se $(t=12.172$; $P<0.001$ ) (Fig. 1).

\subsection{Antennal responses of $P$. xylostella males to conspecific and heterospecific sex pheromone components}

Comparing antennal responses to different doses of the components of the intraspecific pheromone (Z11-16:OAC, Z11-16:OAC and $\mathrm{Z} 11-16: \mathrm{OH})$ and to the components of the two noctuid

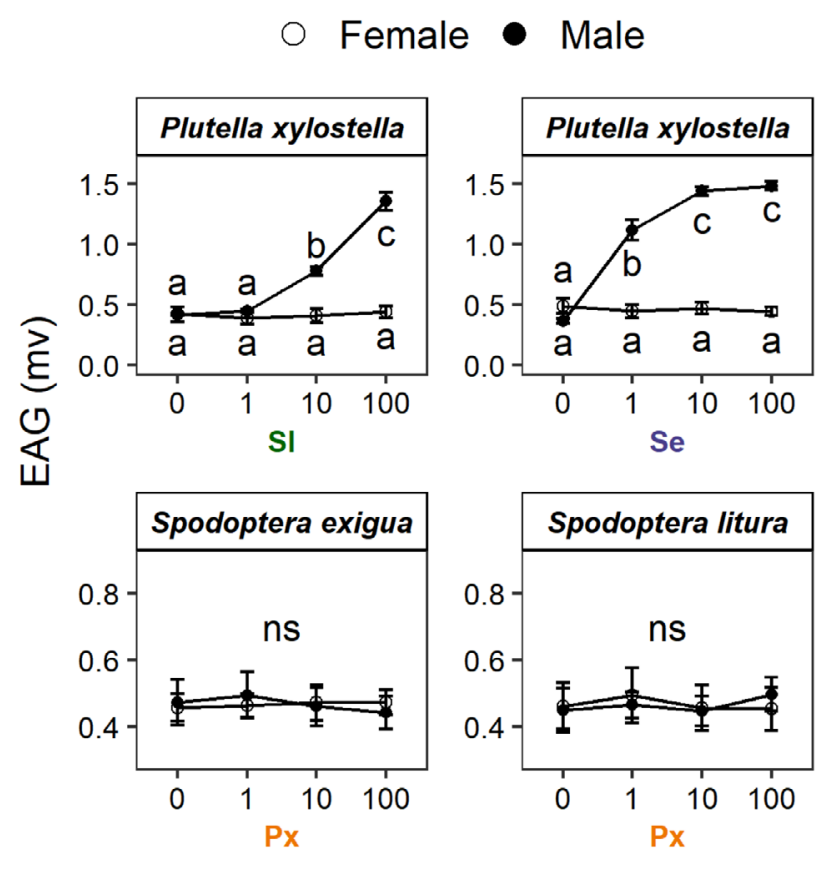

Dose $(\mu \mathrm{g})$

Figure 1. Electrophysiological responses (mean $\pm \mathrm{SE}, \mathrm{mV}$ ) in female and male Spodoptera litura, Spodoptera exigua and Plutella xyloslella. The species names indicate the source of the antennae tested, and the pheromone blends are: SI (S. litura): ZE-9,11-14:OAC and ZE-9,12-14:OAC (90:10 $\mathrm{W} / \mathrm{w})$. Se (S. exigua): ZE-9,12-14:OAc and Z9-14:OH (90:10 w/w), Px (P. xyloslella): Z11-16:OAc, Z11-16:Ald and Z11-16:OH (27:64:9 w/w). Statistical differences across doses for each sex and species are indicated by different letters (ANOVA and Tukey's HSD, $P<0.05$, ns, not significant). $N=6$ antennae per mean.

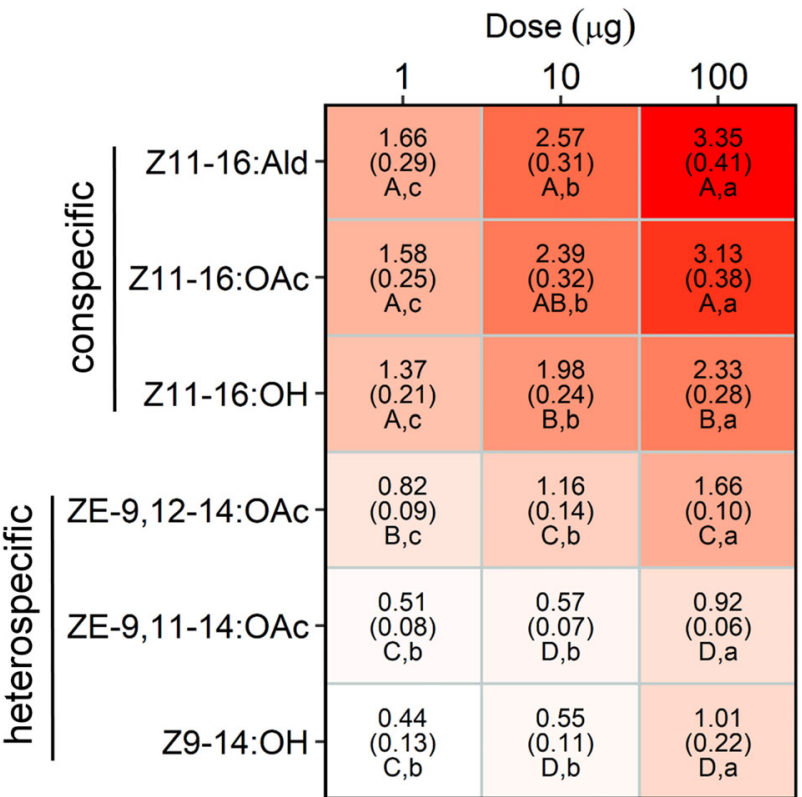

Figure 2. Electrophysiological responses (mean $\pm \mathrm{SE}, \mathrm{mV}$ ) in male moths of Plutella xyloslella to conspecific and heterospecific pheromone components at three different doses. The heat map illustrates the gradient of responses with the greatest responses to conspecific components. Different capital letters within each column indicate significant differences among pheromone components, while different lower-case letters within each row indicate significant differences among the doses (Tukey's HSD, $P<0.05)$. $\mathrm{N}=6$ antennae per mean. 
species (ZE-9,11-14:OAC, ZE-9,12-14:OAc, and Z9-14:OH), we found no significant interaction between 'component' and 'dose' $\left(F_{10,90}=1.731, P=0.086\right)$ (Fig. 2$)$, suggesting that the response to different doses did not differ significantly among the pheromone components. Within each of the dose levels, antennal responses to the three conspecific pheromone components were significantly greater than to each of the noctuid pheromone components, and responses to ZE-9,12-14:OAC were significantly greater than to the other two noctuid pheromone components $(P<0.05)$. On the other hand, antennal responses to the conspecific pheromone components and ZE-9,12-14:OAc increased significantly with increasing doses $(P<0.05)$, while the responses to ZE-9,12-14:OAC and to Z9-14:OH increased significantly only at a dose of $100 \mu \mathrm{g}(P<0.05)$.

\subsection{The noctuid moth sex pheromones antagonize the orientation of $\boldsymbol{P}$. xylostella in a wind tunnel}

In the wind tunnel, $80.00 \%$ of tested $P$. xylostella males activated in response to pheromone blend $\mathrm{Px}$ and $55.00 \%$ of tested males eventually reached the odor source (Table 2). When Z9-14OH and pheromone blend Px were presented together, the percentages of all behavioral categories decreased slightly, but not significantly $(P>0.05)$. Pheromone odor treatment had significant effects on males' orientated flight halfway $\left(H T: \chi^{2}=12.179\right.$, $d f=5, P=0.032)$, approaching within $15 \mathrm{~cm}$ of the source (AP: $\left.\chi^{2}=19.763, d f=5, P=0.001\right)$ and source contact (SC: $\left.\chi^{2}=23.398, d f=5, P<0.001\right)$. The percentage of males that oriented halfway up the wind tunnel $(H T)$ significantly decreased when pheromone blend $\mathrm{Px}$ was combined with blend $\mathrm{SI}$ $(z=3.133, P=0.021)$. The percentage of males flying close to source $(A P)$ significantly decreased when pheromone blend $P x$ was mixed with either blend $\mathrm{SI}(z=3.401, P=0.009)$, blend Se $(z=3.032, P=0.0293)$, or ZE-9,11-14:OAC $(z=3.169$, $P=0.013)$, while the reduction was marginally significant when pheromone blend Px was mixed with ZE-9,12-14:OAc $(z=2.598$, $P=0.098)$. The percentage of males that contacted the odor source (SC) significantly decreased when pheromone blend Px was combined with either blend SI $(z=3.741, P=0.003)$, blend

Table 2. Behavioral responses of Plutella xylostella males to various pheromone sources in a wind tunnel

\begin{tabular}{|c|c|c|c|c|c|}
\hline \multirow[b]{2}{*}{ Odor source ${ }^{\dagger}$} & \multirow[b]{2}{*}{ Males $^{\ddagger}$} & \multicolumn{4}{|c|}{ Male response $(\%)^{\S}$} \\
\hline & & TO & $H T$ & $A P$ & SC \\
\hline Hexane & 34 & 17.65 & 5.88 & 2.94 & 0 \\
\hline Px & 60 & 80.00 & $66.67 \mathrm{~b}$ & $61.67 \mathrm{~b}$ & $55.00 \mathrm{~b}$ \\
\hline$P x+S I$ & 52 & 65.38 & $38.46 \mathrm{a}$ & $28.85 \mathrm{a}$ & $19.23 \mathrm{a}$ \\
\hline $\mathrm{Px}+\mathrm{Se}$ & 62 & 77.42 & $53.23 \mathrm{ab}$ & $33.87 \mathrm{a}$ & $25.81 \mathrm{a}$ \\
\hline$P x+Z E-9,11-14: O A C$ & 59 & 74.58 & $47.46 \mathrm{ab}$ & $32.23 \mathrm{a}$ & $27.12 \mathrm{a}$ \\
\hline$P X+Z E-9,12-14: O A C$ & 54 & 77.78 & $50.00 \mathrm{ab}$ & $37.04 \mathrm{ab}$ & $29.63 \mathrm{ab}$ \\
\hline $\mathrm{Px}+\mathrm{Z9}-14: \mathrm{OH}$ & 50 & 70.00 & $60.00 \mathrm{ab}$ & $52.00 \mathrm{ab}$ & $46.00 \mathrm{ab}$ \\
\hline \multicolumn{6}{|c|}{ 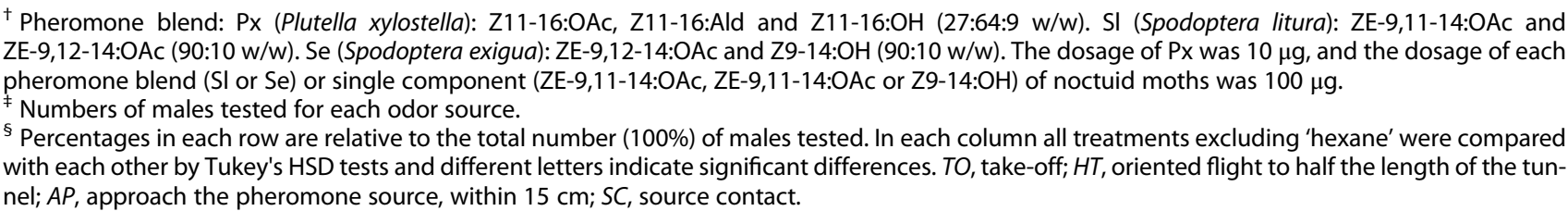 } \\
\hline
\end{tabular}

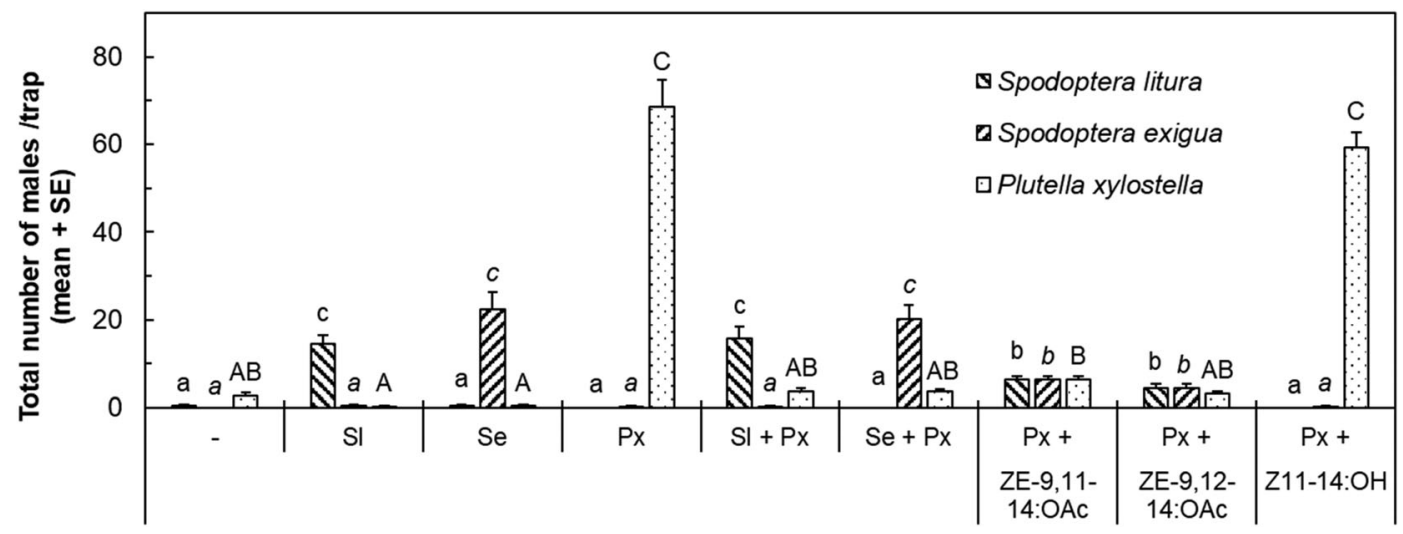

Figure 3. Field trapping of males of Spodoptera litura, Spodoptera exigua and Plutella xyloslella by their independent or combined sex pheromone lures. This study was conducted from 26 May to 3 June 2013 in a cabbage (Brassica oleracea L.) field in Taizhou City, China. The pheromone blends were: SI (S. litura): ZE-9,11-14:OAc and ZE-9,12-14:OAc (90:10 w/w). Se (S. exigua): ZE-9,12-14:OAc and Z9-14:OH (90:10 w/w), Px (P. xyloslella): Z11-16:OAc, Z11-16:Ald and Z11-16:OH (27:64:9 w/w). Doses of lures were $10 \mu \mathrm{g}$ for Px and $100 \mu \mathrm{g}$ for Se and SI. For each species total captures of males ( $\mathrm{n}=6 \mathrm{traps})$ were compared by ANOVA and Tukey's HSD $(\alpha=0.05)$ after $\log _{10}(x+1)$ transformation and statistical differences are represented by lowercase, italics, and capital letters, respectively. 
Se $(z=3.228, P=0.016), \mathrm{ZE}-9,11-14: \mathrm{OAc}(z=3.040, P=0.029)$, while the reduction was marginally significant when pheromone blend Px was mixed with ZE-9,12-14:OAc ( $Z=2.697$, $P=0.076)$

\subsection{Noctuid pheromones reduced catches of $P$. xylostella in the field}

In the first cabbage field, sex pheromone lures of $P$. xylostella were combined with sex pheromone lures of the noctuid moths (Fig. 3). The results showed that numbers of $S$. litura males captured did not differ significantly between traps baited with pheromone blend $\mathrm{SI}$ alone and with $\mathrm{SI}+\mathrm{Px}(z=0.570, P=0.990)$. Likewise, catches of $S$. exigua males were not significantly different between the traps baited with pheromone blend Se alone and with Se $+\operatorname{Px}(z=0.754, P=0.998)$. However, compared to catches of $P$. xylostella in Px traps, significantly fewer $P$. xylostella males were captured when pheromone blend $P x$ was combined with SI $(z=12.276, P<0.001)$, Se $(z=12.189, P<0.001), Z \mathrm{E}-9,11-14$ : OAC $(z=12.833, P<0.001)$ or $Z E-9,12-14:$ OAC $(z=11.984$, $P<0.001)$, but not when $P x$ was combined with $Z 9-14: O H$ $(z=1.858, P=0.643)$.

\subsection{The noctuid moth sex pheromones reduced catches of $P$. xylostella in a dose-dependent manner}

In the cauliflower field, the attraction of $P$. xylostella was examined in traps baited with lures with Px pheromone and with different doses $(1-100 \mu \mathrm{g})$ of sex pheromone blends and single components of the two noctuid species (Fig. 4). Overall, adding 20-

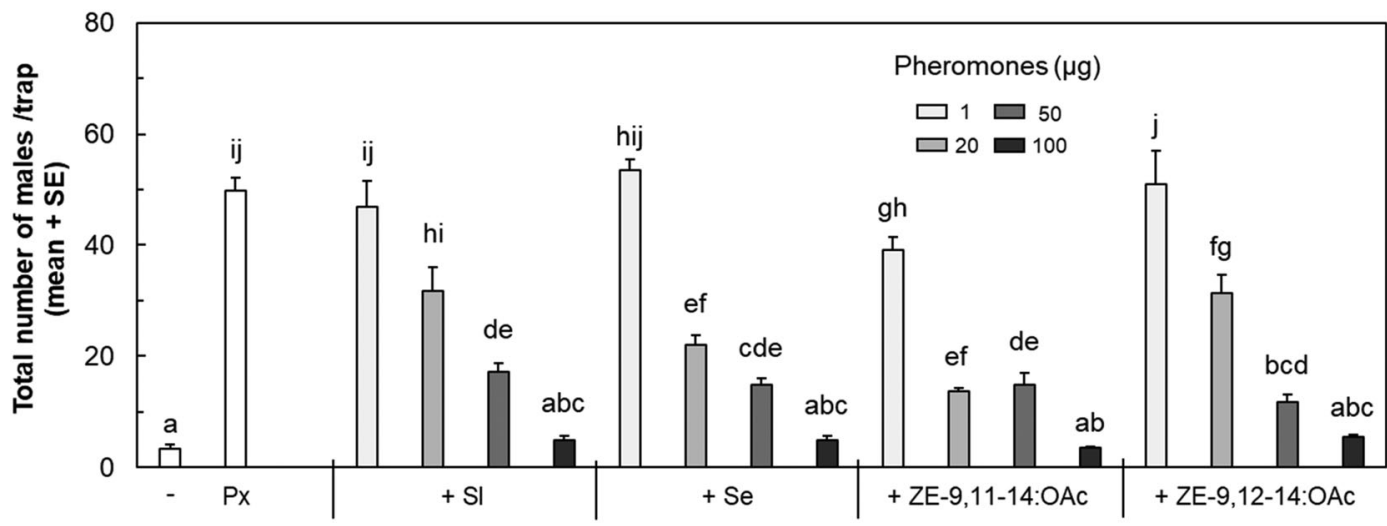

Figure 4. Field trapping of Plutella xylostella males by Px lures plus different doses of other noctuid pheromones. This study was conducted from 4-12 June 2014 in a cauliflower (Brassica oleracea var. botrytis) field in Shanghai, China. The pheromone blends were: SI (Spodoptera litura): ZE-9,11-14:OAc and ZE-9,12-14:OAc (90:10 w/w). Se (Spodoptera exigua): ZE-9,12-14:OAc and Z9-14:OH (90:10 w/w), Px (P. xyloslella): Z11-16:OAc, Z11-16:Ald and Z11-16:OH $(27: 64: 9 \mathrm{w} / \mathrm{w})$. Different letters indicate significant differences of total captures of males which were compared after $\log _{10}(x+1)$ transformation $(n=6$, mean $+\mathrm{SE}$, ANOVA and Tukey's HSD, $P<0.05)$.

A

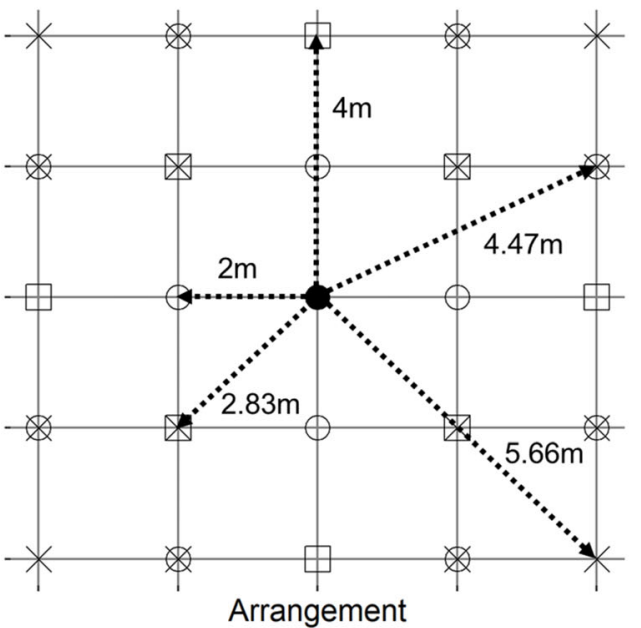

B
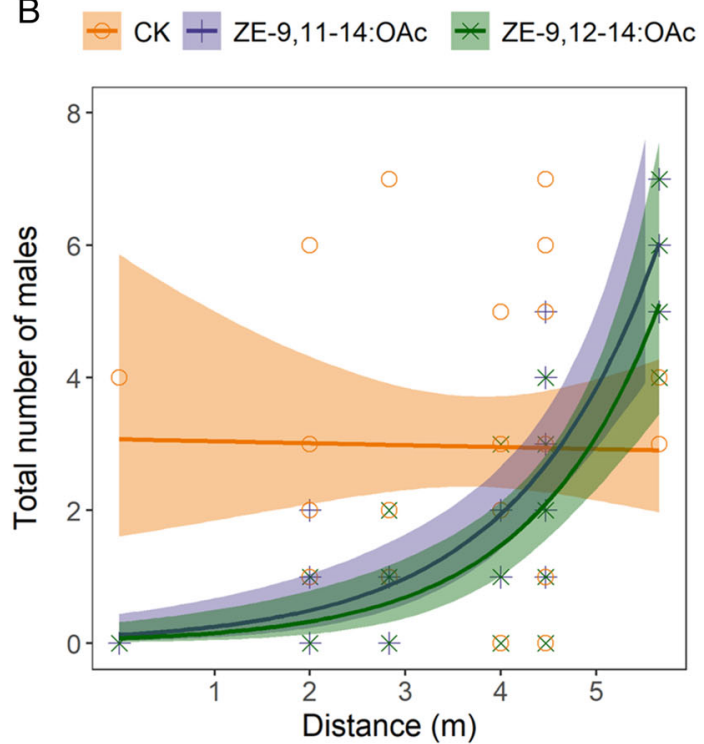

Figure 5. Catches of Plutella xylostella males by $P$. xylostella $(\mathrm{Px})$ lures at varying distances from a central Px lure (P. xyloslella: Z11-16:OAc, Z11-16:Ald and $\mathrm{Z11}-16: \mathrm{OH}, 27: 64: 9 \mathrm{w} / \mathrm{w}$ ) that also contained either ZE-9,11-14:OAc or ZE-9,12-14:OAc. This study was conducted from 22-28 October 2017 in a cabbage (Brassica oleracea L.) field in Pinghu County, Zhejiang, China. (A) Arrangement of the 25 traps baited with various lures. A central trap contained the Px lure, and either ZE-9,11-14:OAC or ZE-9,12-14:OAc and traps at various distances from the central trap contained only the Px lure. (B) The effects of distance (in $\mathrm{m}$ ) to the central lure (Px plus either ZE-9,11-14:OAc or ZE-9,12-14:OAC) on catches of males in traps baited with only the Px lure. Data were fitted by Poisson models and the shaded areas indicate $95 \% \mathrm{Cl}$. $P$ value was from the one sample $z$-test for the coefficient of 'distance' in each regression model (Control (CK): $-0.0098 \pm 0.083, z=-0.118, P=0.91$; ZE-9,11-14:OAc: $0.68 \pm 0.13, z=5.14, P<0.001 ; \mathrm{ZE}-9,12-14: \mathrm{OAc}: 0.75 \pm 0.15, z=4.87, P<0.001$ ). 
$100 \mu \mathrm{g}$ of either pheromone blend of the two Spodoptera species or each of the two single components (ZE-9,11-14:OAc or ZE9,12-14:OAc), significantly reduced the catches of $P$. xylostella males (Tukey's HSD, $P<0.05$ ). Adding $100 \mu \mathrm{g}$ of each of these pheromone treatments to Px reduced the catches of $P$. xylostella to levels that were not significantly different from the blank negative control lure (Tukey's HSD, $P>0.05$ ).

\subsection{Proximity of noctuid pheromones to P. xylostella pheromone lures affects trap catch of $\boldsymbol{P}$. xylostella males}

We positioned traps containing Px lures at various distances from a central trap that also contained noctuid lures to assess the spatial range of noctuid inhibition on catches of $P$. xylostella males (Fig. 5). The effect of distance on total captures of $P$. xylostella males was analyzed in each Poisson model by estimating the coefficient of the 'distance' term. When a Px pheromone lure and a control (hexane, CK) lure were positioned together in the central trap, the total $P$. xylostella males trapped at different distances were not significantly different (CK model: AIC $=109.32$, distance coefficient $=-0.010 \pm 0.083, z=-0.118, P=0.906$ ). However, when the central trap had a Px pheromone lure as well as a lure that contained $100 \mu \mathrm{g}$ of either ZE-9,11-14:OAc or ZE9,12-14:OAc, there was a significant decline in trap catch with greater proximity to the center trap, meaning that the distance coefficient was significantly greater than zero (ZE-9,11-14:OAC model: AIC $=76.108$, distance coefficient $=0.683 \pm 0.133$, $z=5.142, P<0.001$; ZE-9,12-14:OAc model: AIC $=72.627$, distance coefficient $=0.749 \pm 0.154, z=4.870, P<0.001$ ).

\section{DISCUSSION}

Adult males of the three species (S. exigua, $S$. litura and $P$. xylostella) were active on the same nights, as evidenced by the numbers of adults of each species captured in traps in the cabbage field (Fig. 3). The synthetic lures of the three species were highly species-specific, as evidenced by the lack of crossattraction of each species to the sex pheromone of the other two species. The addition of the sex pheromone of $P$. xylostella $(\mathrm{Px})$ to the species-specific noctuid lures did not affect the number of males of the two Spodoptera species captured (Fig. 3). These findings are consistent with the electrophysiological recordings, showing that the Px pheromone did not elicit antennal responses in males of both noctuid moth species (Fig. 1). On the other hand, ZE-9,11-14:OAc, ZE-9,12-14:OAc as well as the two noctuid pheromone blends significantly reduced numbers of $P$. xylostella males captured in traps baited with the Px species-specific pheromone lure; the noctuid moth sex pheromone compounds reduced numbers of males of $P$. xylostella males captured to the numbers captured in blank control traps in the cauliflower field (Fig. 4). In the wind tunnel bioassay, the approach of $P$. xylostella males to Px pheromone was inhibited by lures containing ZE-9,11-14:OAC or ZE-9,12-14:OAc, with the former component being more effective at inhibiting upwind orientation (Table 2). In another cabbage field (B. oleracea), captures of $P$. xylostella were affected similarly by the distance of the Px lures from a lure that contained $\mathrm{Px}$ as well as ZE-9,11-14:OAC and ZE-9,12-14:OAC (Fig. 5). Although ZE9,11-14:OAC and ZE-9,11-14:OAC were equally inhibitory in the cauliflower field, and ZE-9,11-14:OAc was a more effective inhibitor of attraction in the wind tunnel, antennal responses to ZE9,12-14:OAc were greater compared to ZE-9,11-14:OAc (Fig. 2). These results highlight that EAG responses do not necessarily predict behavioral responses. In addition, differential volatilities of the pheromone components and variation in environmental conditions in different Brassica fields likely affected the performance of these semiochemicals. Nevertheless, our behavioral assays in the wind tunnel were consistent with the electrophysiological recordings; antennal responses and behavioral antagonism were found only in $P$. xylostella males, whereas males of both noctuid moth species were not influenced electrophysiologically nor behaviorally by the sex pheromone of $P$. xylostella.

Unlike the uni-directional behavior inhibition we presented here, pheromone inhibition was mutual between two sympatric corn borer species Ostrinia nubilalis (Lepidoptera: Pyralidae) and Sesamia nonagrioides (Lepidoptera: Noctuidae), despite being in different families and having completely different pheromone components. ${ }^{3,13}$ Since EAG responses elicited by inter- and intraspecific pheromones were comparable in these two species, the authors speculated that antennal responses to the pheromone of the other species were due at least in part to olfactory receptors tuned specifically to these molecules. ${ }^{24}$ In our EAG recording, the three noctuid pheromone components elicited different antennal responses, but all responses were significantly lower than the responses elicited by each of the conspecific pheromones when three dosage levels were tested (Fig. 2). In follow-up studies, it will be important to test more dosage levels and estimate parameters such as threshold dose and saturation dose from the doseresponse curves. This would allow us to better differentiate the antennal responses to conspecific and heterospecific pheromone compounds.

The uni-directional pheromone inhibition in sympatric moths that use different pheromones also occurred between species in related genera (e.g., Heliothis (= Chloridea) virescens and Helicoverpa gelotopoeon), ${ }^{11}$ and in related subfamilies (e.g., Cydia pomonella and Adoxophyes orana, ${ }^{4}$ and Tetanolita mynesalis and Lacinipolia renigera ${ }^{12}$ ). The two sex pheromone components of A. orana are 14-carbon acetate esters, whereas the main sex pheromone component of $C$. pomonella is a 12-carbon alcohol. Yet adding the $A$. orana pheromone to a $C$. pomonella pheromone source resulted in significant inhibition of attraction of male C. pomonella. ${ }^{4}$ This interspecific interaction was further hypothesized to be related to evolutionary divergence in tortricid moths: C. pomonella as well as other Olethreutinae species have evolved to utilize 12-carbon sex pheromones while conspecific males retained olfactory receptors tuned to 14-carbon compounds for interspecific recognition. ${ }^{4}$

Previous research showed that both males and females of $P$. xylostella can detect a broad range of plant volatiles, but only males respond to female sex pheromone components. ${ }^{25}$ Taken together with our results, it is reasonable to speculate two olfactory mechanisms for the cross-species inhibition. The first is that the olfactory receptors in male $P$. xylostella antennae that respond to the three heterospecific pheromone components are also broadly tuned to a range of moth pheromone components. This speculation is supported by evidence from heterologous co-expression analysis-olfactory receptor PxylOR4, which is exclusively expressed in males of $P$. xylostella, responded not only to ZE-9,12-14:OAC, but also with less sensitivity to Z9-14:OAC, a pheromone component used by many moth species. ${ }^{26,27}$ The structural similarity between ZE-9,12-14:OAc, ZE-9,11-14:OAc and Z9-14:OAC also supports this hypothesis, as large amounts of Z9-14:OAC are antagonistic to pheromone attraction of P. xylostella. ${ }^{28,29}$ The second hypothesis is that sensilla on $P$. xylostella male antennae house pheromone receptors that uniquely respond to the noctuid pheromones and not to the 
P. xylostella pheromone components. These receptors might serve important functions in the native habitat of $P$. xylostella, or they might represent relics of evolutionary divergence from a noctuid-like ancestor. The antagonistic effects of ZE-9,12-14:OAC and ZE-9,11-14:OAc can be understood better if more heterospecific pheromones with varied structural relations to conspecific pheromones are tested at both antennal and behavioral levels. Single-sensillum recordings are also essential to compare response profiles of pheromone receptor neurons to conspecific and heterospecific pheromones by dose-response analysis. Differentiation of these two hypotheses will need to await future heterologous expression of candidate pheromone receptors. Nevertheless, in the near-term, cross-habituation studies of P. xylostella male antennae with the conspecific and heterospecific components can provide leads as to whether they stimulate the same broadly tuned receptors or different receptors.

All three of these species exhibit nocturnal reproductive activities, including nocturnal calling behaviors of females and flight responses of males. ${ }^{30,31}$ The circadian rhythms of sexual activities of the two noctuid moths were not constant under different labcontrolled photoperiods and temperatures, ${ }^{30}$ making it difficult to infer the degree of overlap of their mating behaviors under common field conditions. However, the directional inhibition of the pheromones of noctuid moths on the $P$. xylostella male response to female sex pheromone suggests additional strategies for the management of these pest species that coexist in Brassica fields. In our trapping experiment conducted in a cauliflower field, the noctuid pheromone blends, as well as their single components, had comparable effects on decreasing trap catches of P. xylostella males (Fig. 4). Our results suggest that the 14-carbon acetate esters could be optimized to disrupt the orientation of $P$. xylostella males to female-produced sex pheromone and prevent successful mating while concurrently disrupting the mating of these two noctuid moth species. Thus, antagonistic and agonistic actions could simultaneously disrupt the sexual communication of all the three species.

\section{ACKNOWLEDGEMENTS}

This work was funded by grants from the Zhejiang Provincial Natural Science Foundation (Y3100384 \& LY19C140005) and Hangzhou Agricultural and Social Development Research Initiative Design Project (20180416A03). The authors are grateful to Wei $\mathrm{Yu}$, Wei-Jie Fu, Ting Tian, Lu Chen and Robert Cline for their support of this work, and DBM editing for professional proofreading service.

\section{CONFLICT OF INTEREST}

The authors declare no conflict of interest.

\section{DATA AVAILABILITY STATEMENT}

The data that support the findings of this study are available from the corresponding author upon reasonable request.

\section{SUPPORTING INFORMATION}

Supporting information may be found in the online version of this article.

\section{REFERENCES}

1 Groot AT, Horovitz JL, Hamilton J, Santangelo RG, Schal C and Gould F, Experimental evidence for interspecific directional selection on moth pheromone communication. Proc Natl Acad Sci U S A 103: 5858-5863 (2006).

2 Groot AT, Dekker T and Heckel DG, The genetic basis of pheromone evolution in moths. Annu Rev Entomol 61:99-117 (2016).

3 Eizaguirre M, Albajes R, Lopez C, Sans A and Gemeno C, Inhibition of pheromone response in Sesamia nonagrioides by the pheromone of the sympatric corn borer, Ostrinia nubilalis. Pest Manag Sci 63: 608-614 (2007)

4 Potting RPJ, Lösel PM and Scherkenbeck J, Spatial discrimination of pheromones and behavioural antagonists by the tortricid moths Cydia pomonella and Adoxophyes orana. J Comp Physiol A 185: 419-425 (1999).

5 Allison JD and Cardé RT, Pheromones: reproductive isolation and evolution in moths, in Pheromone Communication in Moths, ed. by Allison JD and Cardé RT. University of California Press, Berkeley, CA, pp. 11-24 (2016).

6 Roelofs WL and Rooney AP, Molecular genetics and evolution of pheromone biosynthesis in Lepidoptera. Proc Natl Acad Sci U S A 100: 9179-9184 (2003).

7 Mazor M and Dunkelblum E, Circadian rhythms of sexual behavior and pheromone titers of two closely related moth species Autographa gamma and Cornutiplusia circumflexa. J Chem Ecol 31:2153-2168 (2005).

8 McElfresh JS and Millar JG, Geographic variation in the pheromone system of the saturniid moth Hemileuca eglanterina. Ecology 82:35053518 (2001).

9 Liang Y, Luo M, Fu X, Zheng L and Wei H, Mating disruption of Chilo suppressalis from sex pheromone of another pyralid rice pest Cnaphalocrocis medinalis (Lepidoptera: Pyralidae). J Insect Sci 20:19 (2020).

10 Yan Q, Liu X, Wang Y, Tang X, Shen Z, Dong S et al., Two sympatric Spodoptera species could mutually recognize sex pheromone components for behavioral isolation. Front Physiol 10:1256 (2019).

11 Juárez ML, Ruiz MJ, Fernández PC, Goane L, Villagrán ME, Arce OEA et al., Communication interference in sympatrically occurring moth species. Entomol Exp Appl 158:25-33 (2016).

12 Haynes KF, Gemeno C, Yeargan KV, Millar JG and Johnson KM, Aggressive chemical mimicry of moth pheromones by a bolas spider: how does this specialist predator attract more than one species of prey? Chemoecology 12:99-105 (2002).

13 Gemeno C, Sans A, López C, Albajes R and Eizaguirre M, Pheromone antagonism in the European corn borer moth Ostrinia nubilalis. $J$ Chem Ecol 32:1071-1084 (2006).

14 Lee S, Lee D-W and Boo KS, Sex pheromone composition of the diamondback moth, Plutella xylostella (L) in Korea. J Asia Pac Entomol 8:243-248 (2005).

15 Deng J, Jiang Y, Wang Z, Jiang J and Du J, Field behavioral responses of male diamondback moth, Plutella xylostella to different components, ratios and dosages of synthetic sex attractant in China. J Zhejiang Univ Agric Life Sci 33:514-518 (2007).

16 Dong $\mathrm{S}$ and Du J, Chemical identification and field tests of sex pheromone of beet armyworm Spodoptera exigua. Acta Phyt Sin 29:19-24 (2002).

17 Sun F, Hu Y and Du J, The sex pheromone communication system of Spodoptera litura (Fabricius). Acta Entomol Sin 45:404-407 (2002).

18 Sun F, Du J and Chen T, The behavioral responses of Spodoptera litura (F.) males to the female sex pheromone in wind tunnel and field trapping tests. Acta Entomol Sin 46:126-130 (2003).

19 Wang F, Deng J, Schal C, Lou Y, Zhou G, Ye B et al., Non-host plant volatiles disrupt sex pheromone communication in a specialist herbivore. Sci Rep 6:32666 (2016).

20 Liu Y and Li B, Developmental interactions between Spodoptera exigua (Noctuidae: Lepidoptera) and its uniparental endoparasitoid, Meteorus pulchricornis (Braconidae: Hymenoptera). Biol Control 38: 264-269 (2006).

21 Chen Q, Li G and Pang Y, A simple artificial diet for mass rearing of some noctuid species. Entomol Knowl 37:325-327 (2000).

22 Mazerolle MJ, AICcmodavg: model selection and multimodel inference based on (Q)AIC(c). R package version 2.3.1. https://cran.r-project. org/web/packages/AICcmodavg/AICcmodavg.pdf (2017).

23 Team RC, R: A Language and Environment for Statistical Computing. R Foundation for Statistical Computing, Vienna (2019).

24 Linn CE, Domingue MJ, Musto CJ, Baker TC and Roelofs WL, Support for (Z)-11-hexadecanal as a pheromone antagonist in Ostrinia nubilalis: 
flight tunnel and single sensillum studies with a New York population. J Chem Ecol 33:909-921 (2007).

25 Wu A, Li X, Yan X, Fan W and Hao C, Electroantennogram responses of Plutella xylostella (L.), to sex pheromone components and host plant volatile semiochemicals. J Appl Entomol 144:396-406 (2020).

26 Sun M, Liu Y, Walker WB, Liu C, Lin K, Gu S et al., Identification and characterization of pheromone receptors and interplay between receptors and pheromone binding proteins in the diamondback moth, Plutella xyllostella. PLoS One 8:e62098 (2013).

27 El-Sayed AM, The Pherobase: Database of Insect Pheromones and Semiochemicals. (2016). http://www.pherobase.com [5 May 2021].
28 Chisholm MD, Steck WF, Underhill EW and Palaniswamy P, Field trapping of diamondback moth Plutella xylostella using an improved four-component sex attractant blend. J Chem Ecol 9:113-118 (1983).

29 Suckling DM, Gibb AR, Daly JM, Rogers DJ and Walker GP, Improving the pheromone lure for diamondback moth. N Z Plant Prot 55: 182-187 (2002).

30 Groot AT, Circadian rhythms of sexual activities in moths: a review. Front Ecol Evol 2:43 (2014).

31 Wang G, Huang $X$, Wei $H$ and Fadamiro HY, Sublethal effects of larval exposure to indoxacarb on reproductive activities of the diamondback moth, Plutella xylostella (L.) (Lepidoptera: Plutellidae). Pest Biochem Phys 101:227-231 (2011). 